This document is the Accepted Manuscript version of a Published Work that appeared in final form in Langmuir, copyright (c) American Chemical Society after peer review and technical editing by the publisher. To access the final edited and published work see http://pubs.acs.org/doi/ipdf/10.1021/la404868e.

\title{
Microscopic origin of the surface tension anomaly of water
}

\author{
Marcello Sega ${ }^{*},{ }^{1,2}$ George Horvai, ${ }^{3,4}$ and Pál Jedlovszky, ${ }^{4,5,6}$ \\ ${ }^{1}$ Department of Physics, University of Rome "Tor Vergata", Italy \\ ${ }^{2}$ University of Vienna, Institut für Computergestützte Biologische Chemie, Austria \\ ${ }^{3}$ MTA-BME Research Group of Technical Analytical Chemistry, Budapest, Hungary \\ ${ }^{4}$ Department of Inorganic and Analytical Chemistry, Budapest University of Technology and Economics, \\ Hungary \\ ${ }^{5}$ EKF Department of Chemistry, Eger, Hungary \\ ${ }^{6}$ Institute of Chemistry, ELTE University, Budapest, Hungary \\ *Electronic mail: marcello.sega@univie.ac.at
}

\begin{abstract}
We investigate the hydrogen bonding percolation threshold of water molecules at the surface of the liquid-vapor interface. We show that the percolation temperature agrees within statistical accuracy with the high-temperature inflection point of the water surface tension. We associate the origin of this surface tension anomaly of water with the sudden breakup of the hydrogen bonding network in the interfacial molecular layer.
\end{abstract}


Keywords: Percolation, water liquid-vapor interface, water anomalies 


\section{Introduction}

The presence of an inflection point in water's surface tension at $530 \mathrm{~K}$ is one of the many unresolved problems that the most abundant liquid on earth still poses. ${ }^{1}$ The surface tension of the coexisting liquid-vapor phase is known experimentally with high accuracy, ${ }^{2}$ yet no connection with the microscopic structure of water has been made to explain this anomaly, mostly because thermal capillary waves prevented so far a straightforward analysis of the structural changes that happen at the liquid-vapor interface. However, recently developed computational techniques allow us now to investigate the very outmost molecular layer of the interface. ${ }^{3,4}$ Here, we analyze the structure of water's surface molecules to investigate the percolation threshold of the surface hydrogen bonded network. We show that the temperature of the percolation threshold coincides with that of the surface tension inflection. This result is remarkably consistent across four different water models, including three-, and four-site ones. Our finding suggests the collapse of the percolating two-dimensional surface network of hydrogen bonds as the microscopic origin of the anomalous inflection point of water's surface tension, and puts an accent on the importance of the analysis of intrinsic surface properties in the understanding of complex phenomena at liquid-gas and liquid-liquid interfaces.

\section{Computer Simulations.}

Recently, some of us showed that for SPC/E water ${ }^{5}$ the surface percolation well precedes the phase mixing at the liquid-vapor interface. ${ }^{6}$ This finding suggested us the existence of a relation between the percolation temperature and the anomalous inflection of water's surface tension. We soon realized that any such a relation should be model-independent to provide a compelling evidence of its existence. Therefore, we planned a large-scale set of molecular dynamics simulations for four different rigid water models, namely, SPC, ${ }^{7} \mathrm{SPC} / \mathrm{E}, \mathrm{TIP} 4 \mathrm{P},{ }^{8}$ and TIP4P $/ 2005,{ }^{9}$ cumulating $1.3 \mu$ s of simulation time using the 
GROMACS 4.5 simulation package. ${ }^{10}$ For each of these water models we performed simulations at $12-17$ different temperatures, ranging from 300 to $500 \mathrm{~K}$, along the liquid vapor coexistence line, using a temperature grid of $10 \mathrm{~K}$ in the vicinity of the percolation threshold. Each simulation was carried out with two systems of different size, containing 1000 and 4000 molecules, respectively. The size of the respective rectangular basic simulation cells were $25 \times 25 \times 150 \AA$ and, $50 \times 50 \times 250 \AA$, the third value corresponding to the macroscopic surface normal axis.

For each system we performed 2 ns long equilibration runs, followed by 10 ns long production runs. To detect the surface layer molecules we used the identification of truly interfacial molecules (ITIM) method, ${ }^{4}$ using a probe-sphere radius of $1.5 \AA$. To exclude vapor phase molecules from the ITIM analysis, we regarded the largest three-dimensional hydrogen bonded cluster of molecules as the liquid phase (see Figure 1). We proceeded further by performing a cluster analysis, restricted to the surface layer only, of the hydrogen bonded molecules.

\section{Results and Discussion}

The detection of the percolation threshold in computer simulation usually suffers from finite size effects: the point at which the network reaches the size of the simulation box is captured instead of the point where the network becomes infinite. We overcome this problem by determining a suitably defined spanning probability in systems of different size. Since the largest cluster at the percolation threshold is a fractal object of well-defined dimension, ${ }^{11}$ the probability that it spans the system is sizeindependent at the percolation threshold. ${ }^{12}$ Here, two molecules are considered to be $\mathrm{H}$-bonded if the distances of the $\mathrm{O}$ atoms and shortest $\mathrm{O}-\mathrm{H}$ pair are smaller than 3.35 and $2.45 \AA$, respectively. ${ }^{13}$ In addition, we define a cluster as an assembly of surface molecules connected by a continuous chain of $\mathrm{H}$ bonds, and the spanning probability $P_{\mathrm{sp}}$ as the probability that the largest cluster spans the surface of the system along both dimensions (calculated as the occurrences of a percolating cluster divided by the 
total number of frames). We determine the percolation threshold as the intersection temperature of the $P_{\text {sp }}(T)$ curves obtained in the small and large systems. ${ }^{14}$

This method allows us also to identify the percolation threshold very precisely, and with low computational effort. In Figure 2 we show $P_{\text {sp }}(T)$ for the different models. The intersection of the two curves is easily identified, and allows us to interpolate linearly the percolation temperature. In Figure 2 we present also the percolation temperature as obtained from the average (asterisks) between the twodimensional spanning probability and an alternative (one-dimensional) spanning probability. The results are compatible within the resolution grid. The reduced percolation temperatures $T_{\mathrm{p}}{ }^{*}=T_{\mathrm{p}} / T_{\mathrm{c}}\left(T_{\mathrm{c}}\right.$ being the critical temperature of the given model) are reported in Table 1. For all models, the reduced percolation temperature is in the range $0.70+/-0.01$.

Locating the inflection point of the surface tension turned out to be a less straightforward task. All the surface tension curves obtained for the large systems, presented in Figure 3, show only a feeble inflection, and hence statistical noise makes it difficult to evaluate it at first sight. Therefore, we decided to extract the position of the inflection using two different fitting functions, namely a Fermi function:

$$
\gamma(T)=\frac{B}{1+\exp \left[C\left(T-T_{\mathrm{i}}\right)\right]}
$$

( $B$ and $C$ being fitting parameters), and a third order polynomial, using as a fitting range the interval from 300 to $500 \mathrm{~K}$. We reported the resulting reduced inflection temperatures $T_{\mathrm{i}}{ }^{*}=T_{\mathrm{i}} / T_{\mathrm{c}}$ in Table 1 and in the inset of Figure 3 . The results of the fits agree surprisingly well for all models and are in the range $0.71+/-0.02$ in every case. The simulated surface tension data and the parameters of their Fermi fit are presented in Tables S1 and S2 in Supporting Information. To demonstrate that the simulated data points indeed show an inflection, we fitted a straight line to the $\lambda(T)$ data, and plotted the difference from their linear fit vs. temperature (see Figure S1 in Supporting Information). This difference shows a pronounced oscillation around zero, crossing it twice in the simulated temperature range for all models. 
Our simulations indicate that for all models, the reduced inflection temperature coincides within statistical accuracy with the percolation threshold's reduced temperature. With this information at hand, we are now able to support the explanation that when the temperature reaches the percolation threshold, the collapse of the hydrogen bonding network at the surface is responsible for the increase in the change of the surface tension, namely, of the inflection. This result parallels in some sense the one for the three-dimensional percolation threshold of supercritical water, which is found to be slightly above the analytical extrapolation of the coexistence line. ${ }^{14,15}$ In other words, along the coexistence line water encounters two points, one at lower temperatures where the two-dimensional connectivity of the surface network of hydrogen bonds is broken, followed by the critical point, where the collapse of the hydrogen bond network extends to the bulk. At a difference with the critical point, the inflection one is influenced only by the connectivity at the surface, as the surface tension is a measure of the energy needed to create surface area. At a difference with the critical point, the inflection one is influenced only by the surface connectivity, since the surface tension is a measure of the energy required to create surface area. The use of an intrinsic analysis is therefore the key to investigate and interpret this phenomenon, as a non-intrinsic analysis would have inevitably taken into account molecules below the surface layer.

At the same time, our simulations show that the inflection temperature or, equivalently, the percolation temperature, is a rather robust feature across different models. We found that this robustness is extended to two other important quantities. First, the two dimensional spanning probability at the percolation threshold, $P_{\mathrm{sp}}\left(T_{\mathrm{p}}\right)$, is always compatible with the value of $0.250+/-0.005$. Second, the distribution of the number of hydrogen bonded neighbors, which we show in Figure 4, is identical for all the different models at the percolation threshold, and shows that the inflection point is characterized by a clear structural fingerprint, which appears to be model-independent. This universal behavior is rooted in the purely geometrical nature of the percolation threshold: the number of 
hydrogen bonded neighbors should not depend on the fine-grain detail of the water models, as long as they support the same type of network topology (This, however, clarifies only one of the two universal behaviors we observed, as we did not find so far an explanation for the apparent universality of the ratio $T_{\mathrm{p}} / T_{\mathrm{c}}$ ). The hydrogen bond formation probability at the percolation threshold is equal to $0.63+/-0.02$ for all the four models considered, assuming (as supported by the low fraction of four-bonded molecules, see Figure 4) that every molecule can form up to three hydrogen bonds with its neighbors in the surface layer. This value agrees surprisingly well with the percolation threshold of the honeycomb lattice of $0.653,{ }^{16}$ suggesting that, similarly to the distorted tetrahedral network of molecules in bulk water, molecules at the surface form a distorted hexagonal network, in a clear analogy with the honeycomb-like arrangement of the molecules at the (0001) cut of the $I_{h}$ ice crystal.

Supporting Information. The simulated surface tension data and the parameters of their fermi fits (with error bars) as well as the deviation of the simulated surface tension data from their linear fit are provided. This information is available free of charge via the Internet at http://pubs.acs.org.

Acknowledgements. The authors acknowledge the Hungarian OTKA Foundation (Project No. 104234), the MTA-CNR bilateral grant, the European Union (FP7 IEF Project No. 331932). P.J. is a Szentágothai János fellow of Hungary, supported by the European Union, co-financed by the European Social Fund in the framework of TÁMOP 4.2.4.A/2-11/1-2012-0001 "National Excellence Program" under grant number A2-SZJÖ-TOK-13-0030.

\section{References}


(1) Rowlinson, J. S.; Widom, B. Molecular theory of Capillarity; Dover Publications: Mineola, 2002,

p. 11.

(2) Vargaftik, N. B.; Volkov, B. N.; Voljak, L. D. International Tables of the Surface Tension of Water. J. Phys. Chem. Ref. Data 1983, 12, 817-820.

(3) Chacón, E.; Tarazona, P. Intrinsic Profiles Beyond the Capillary Wave Theory: A Monte Carlo Study. Phys Rev. Lett. 2003, 91, 166103.

(4) Pártay, L. B.; Hantal, Gy.; Jedlovszky, P.; Vincze, Á.; Horvai, G. A New Method for Determining the Interfacial Molecules and Characterizing the Surface Roughness in Computer Simulations. Application to the Liquid-Vapor Interface of Water. J. Comp. Chem. 2008, 29, 945-956.

(5) Berendsen, H. J. C.; Grigera, J. R.; Straatsma, T. P. The Missing Term in Effective Pair Potentials. J. Phys. Chem.1987, 91, 6269-6271.

(6) Darvas, M.; Horvai, G.; Jedlovszky, P. Temperature Dependence of the Lateral Hydrogen Bonded Clusters of Molecules at the Free Water Surface. J. Mol. Liq. 2012, 176, 33-38.

(7) Berendsen, H. J. C.; Postma, J. P. M.; van Gunsteren, W. F.; Hermans, J. Interaction Models for Water in Relation to Protein Hydration. In Intermolecular Forces, Pullman, B., Ed., Reidel: Dordrecht, 1981, p. 331-342.

(8) Jorgensen, W. L.; Chandrashekar, J.; Madura, J. D.; Impey, R.; Klein, M. L. Comparison of Simple Potential Functions for Simulating Liquid Water. J. Chem. Phys. 1983, 79, 926-935.

(9) Abascal, J. L. F.; Vega, C. A General Purpose Model for the Condensed Phases of Water: TIP4P/2005. J. Chem. Phys. 2005, 123, 234505. 
(10) Hess, B.; Kutzner, C.; van der Spoel, D.; Lindahl, E. GROMACS 4: Algorithms for Highly Efficient, Load-Balanced, and Scalable Molecular Simulation. J. Chem. Theory Comput. 2008, 4, 435-447.

(11) Stauffer, D. Introduction to percolation theory; Taylor and Francis: London, 1985.

(12) Hovi, J. P.; Aharony, A. Scaling and universality in the spanning probability for percolation. Phys. Rev. E 1996, 53, 235-253.

(13) Jedlovszky, P.; Brodholt, J. P.; Bruni, F.; Ricci, M. A.; Soper, A. K.; Vallauri, R. Analysis of the hydrogen-bonded structure of water from ambient to supercritical conditions. J. Chem. Phys. 1998, 108, 8528-8540.

(14) Skvor, J.; Nezbeda, I.; Brovchenko, I.; Oleinikova, A. Percolation Transition in Fluids: Scaling Behavior of the Spanning Probability Functions. Phys. Rev. Lett. 2007, 99, 127801.

(15) Pártay, L.; Jedlovszky, P. Line of Percolation in Supercritical water. J. Chem. Phys. 2005, 123, 024502.

(16) Sykes, M. F.; Essam, J. W. Exact critical percolation probabilities for site and bond problems in two dimensions. J. Math. Phys. 1964, 5, 1117-1127.

(17) de Pablo, J. J.; Prausnitz, J. M.; Strauch, H. J.; Cummings, P. T. Molecular Simulation of Water along the Liquid-Vapor Coexistence Curve from $25^{\circ} \mathrm{C}$ to the Critical Point. J. Chem. Phys. 1990, 93, 73557359.

(18) Guissani, Y.; Guillot, B. A Computer Simulation Study of the Liquid-Vapor Coexistence Curve of Water. J. Chem. Phys. 1993, 98, 8221-8235.

(19) Lísal, M.; Nezbeda, I.; Smith, W. R. Vapor-Liquid Equilibria in Five-Site (TIP5P) Models of Water. J. Phys. Chem. B 2004, 108, 7412-7414. 
(20) Vega, C.; Abascal, J. L. F., Nezbeda, I. Vapor-Liquid Equilibria from the Triple Point up to the Critical Point for the New Generation of TIP4P-like Models: TIP4P/Ew, TIP4P/2005, and TIP4P/ice. J. Chem. Phys. 2006, 125, 034503. 
Table 1. Temperature of surface percolation and surface tension inflection of the four water models, normalized by the critical temperature, $T_{c}$. The critical temperature values are also shown.

\begin{tabular}{|c|c|c|c|c|}
\hline \multirow[b]{2}{*}{ Water model } & \multicolumn{4}{|c|}{$\overline{T_{\mathrm{i}}^{*}}$} \\
\hline & $T_{\mathrm{p}}^{*}$ & $\begin{array}{l}\text { fitting Fermi } \\
\text { function }\end{array}$ & $\begin{array}{c}\text { fitting } 3^{\text {rd }} \text { order } \\
\text { polynomial }\end{array}$ & $T_{\mathrm{c}} / \mathrm{K}$ \\
\hline SPC & $0.707 \pm 0.009$ & $0.707 \pm 0.012$ & $0.726 \pm 0.009$ & $587^{a}$ \\
\hline SPC/E & $0.698 \pm 0.008$ & $0.687 \pm 0.014$ & $0.709 \pm 0.008$ & $652^{\mathrm{b}}$ \\
\hline TIP4P & $0.692 \pm 0.005$ & $0.702 \pm 0.009$ & $0.709 \pm 0.005$ & $588^{c}$ \\
\hline TIP4P/2005 & $0.697 \pm 0.008$ & $0.705 \pm 0.006$ & $0.717 \pm 0.008$ & $640^{d}$ \\
\hline
\end{tabular}



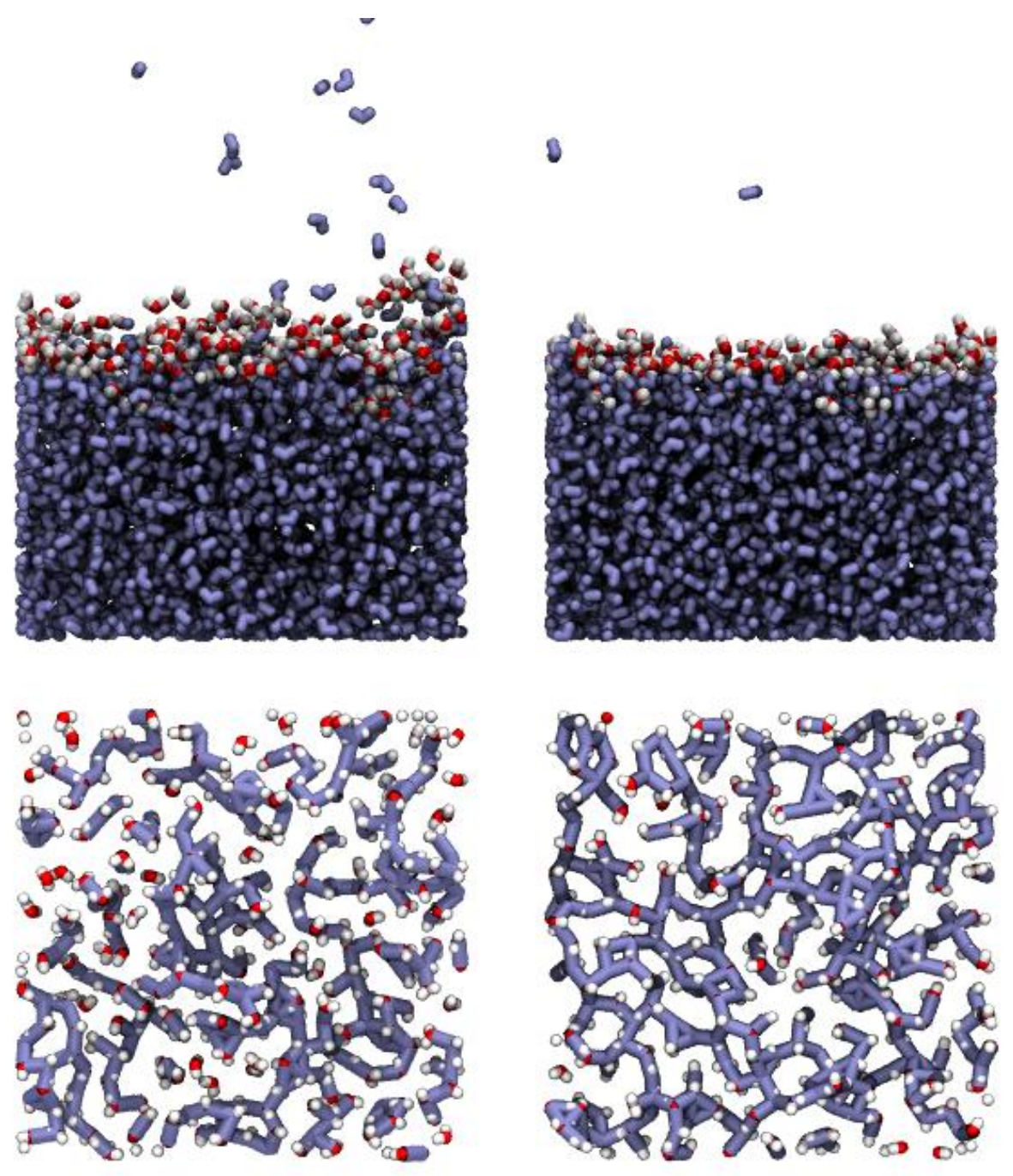

Figure 1. Detail of the water-vapor interface for the SPC model. Top row: above ( $450 \mathrm{~K}$, left) and below (300 K, right) the inflection point. Molecules not belonging to the first liquid layer are shown in blue. Bottom row: top view of the surface layer. The connectivity network between O atoms within a $3.35 \AA$ cutoff distance is marked using blue bonds ( $450 \mathrm{~K}$, left and $300 \mathrm{~K}$, right). 


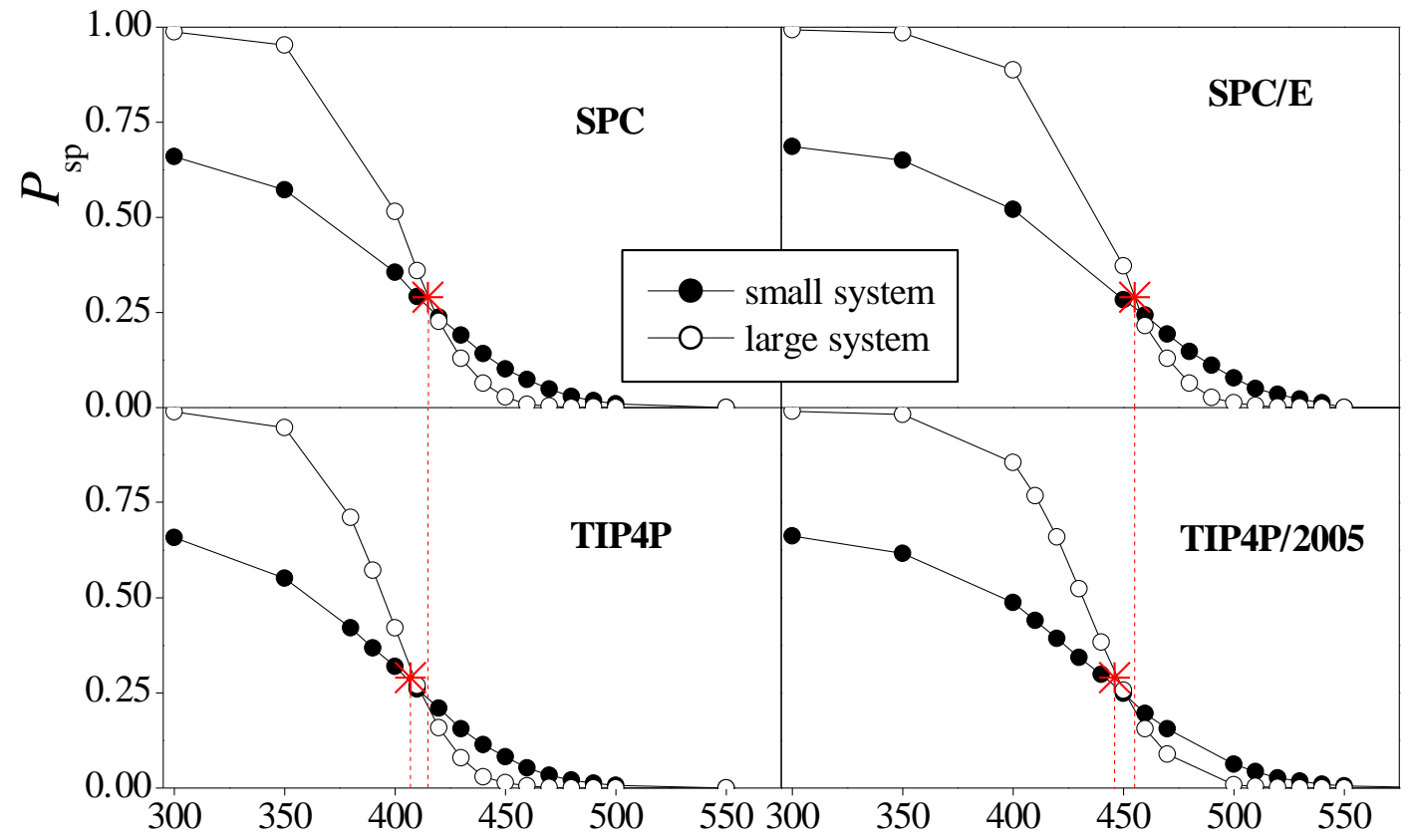

Figure 2. Spanning probability in the surface layer. Red asterisks mark the surface percolation threshold. 


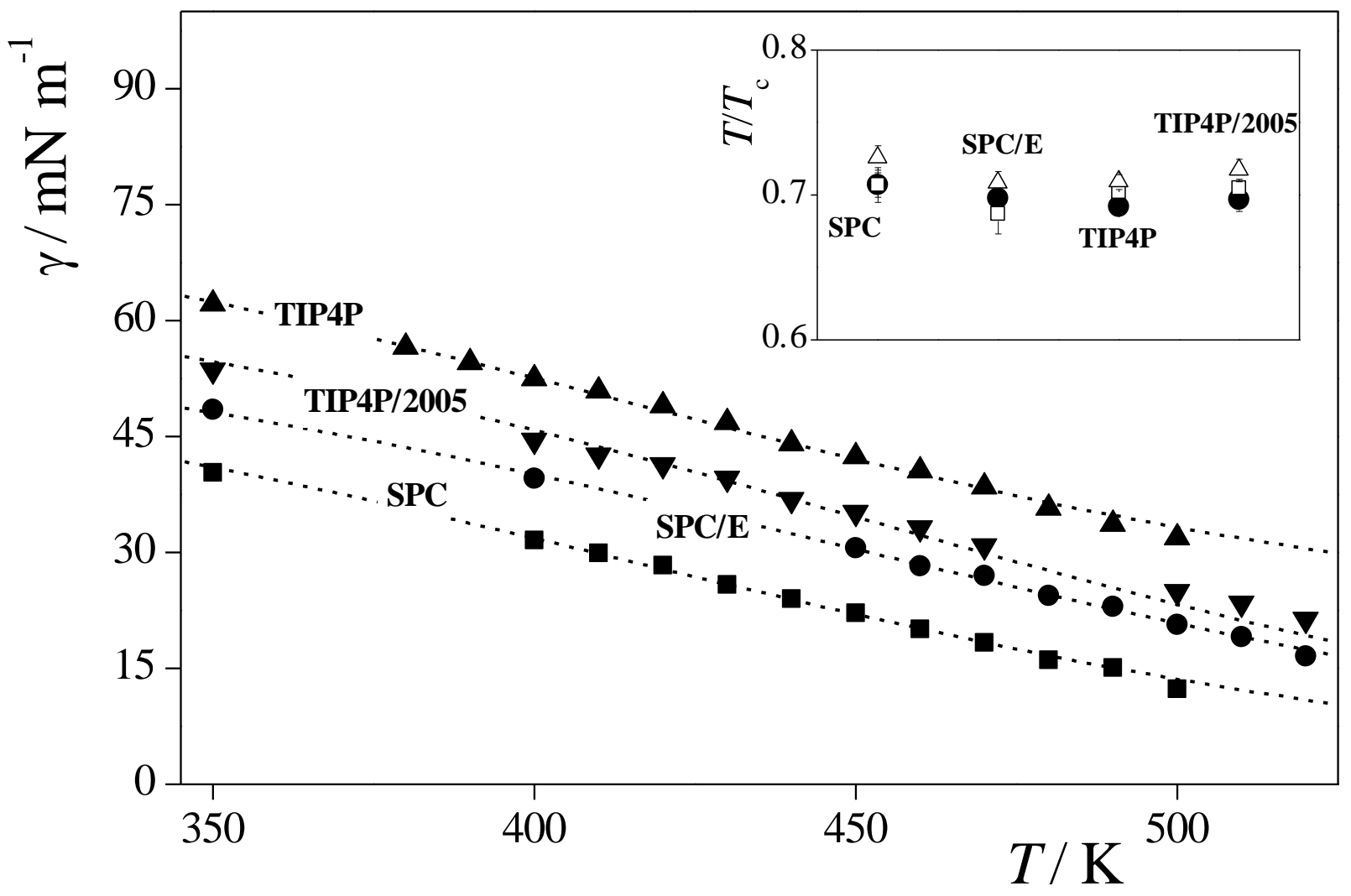

Figure 3. Surface tension of the four models. Dashed lines: Fermi function resulting from the best fit.

The TIP4P data are shifted up by $20 \mathrm{mN} / \mathrm{m}$. The standard deviation is always less than $0.5 \mathrm{~g} / \mathrm{mN} / \mathrm{m}$. Inset: reduced surface percolation temperature (full circles) and reduced surface tension inflection temperature obtained from the Fermi function fit (open squares) from the polynomial fit (open triangles). 


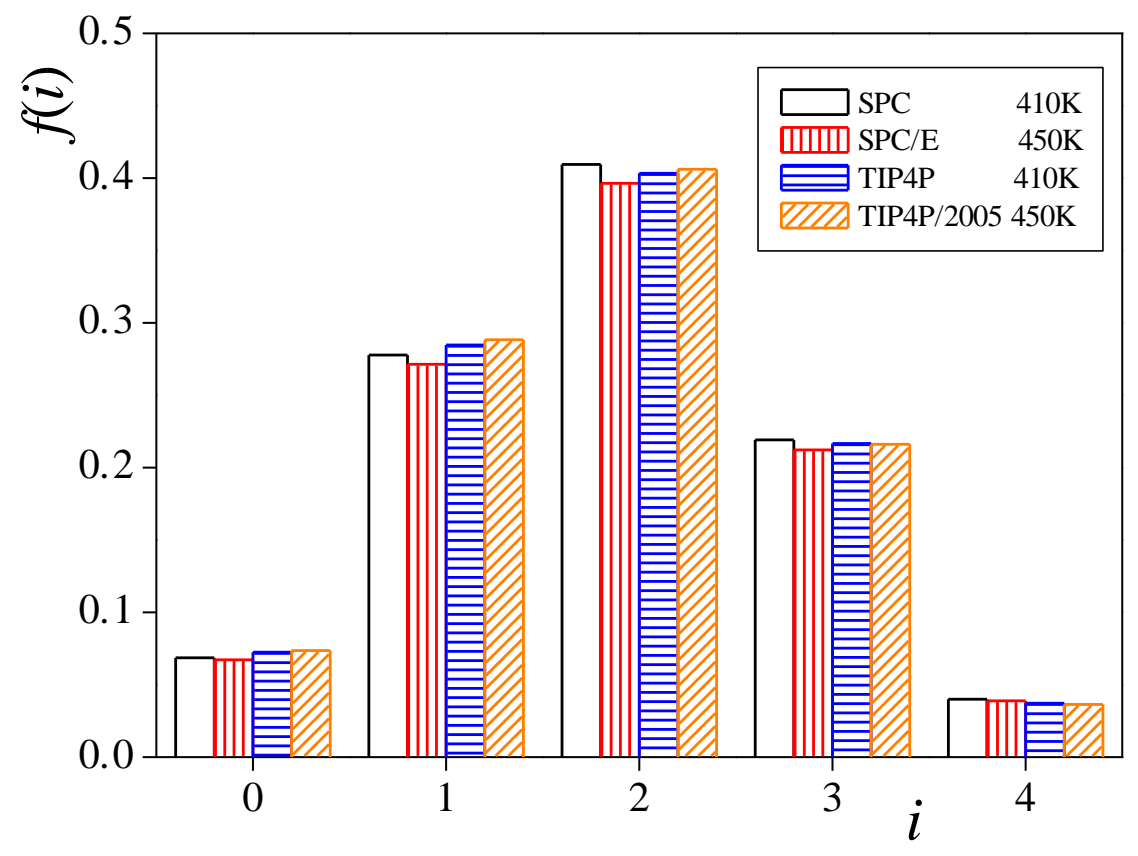

Figure 4. Fraction of the surface water molecules having $i$ hydrogen bonding neighbors within the surface layer. The statistics is calculated at the surface percolation threshold 
TOC GRAPHICS
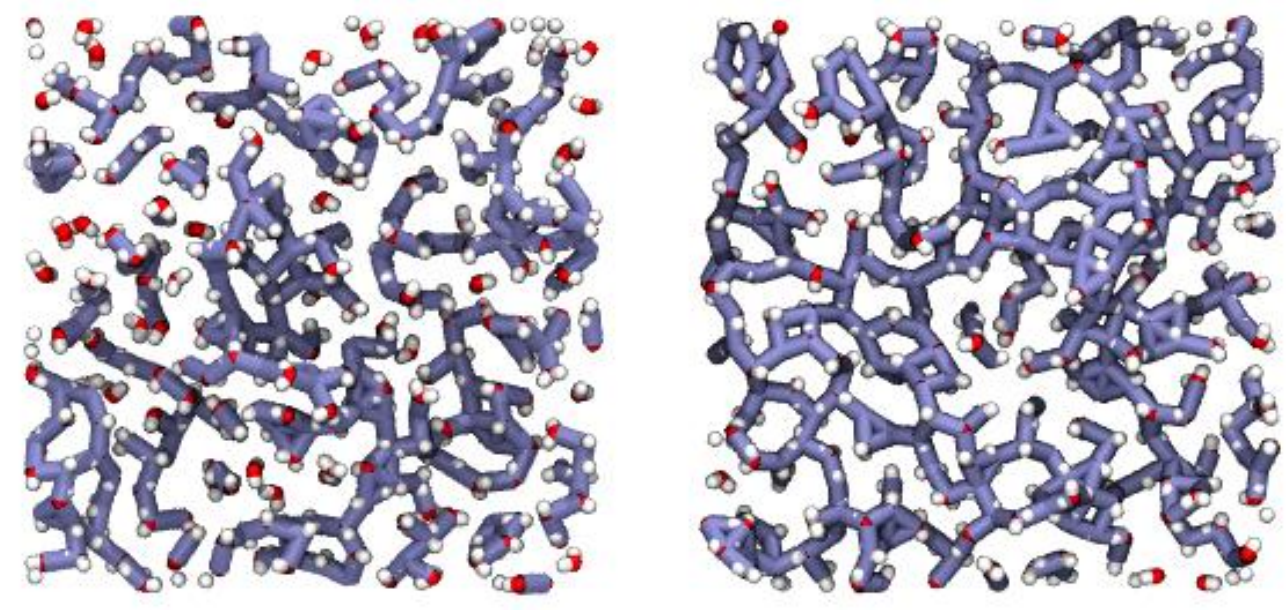